\title{
DEBITMETRE AUXILIAIRE EN ANESTHESIE OBSTETRICALE
}

\author{
L. Perras, M.D., et C. Leduc, M.D.*
}

Au couns de l'anesthésie obstétricale, l'anesthésiste se trouve parfois confronté avec deux patients en même temps; la mère et le nouveau-né, les problèmes de la réanimation ajoutent un lourd fardeau d l'exercice de son art.

Pendant la nuit ou lorsque l'activité intense des salles d'opération ne permet pas d'obtenir d'urgence l'aide d'un confrère expérimenté, l'anesthésiste doit alors se tirer d'embarras avec les moyens du bord.

Les appareils de réanimation automatiques dont nous disposons ont des limites d'efficacité. L'oxygénation au moyen d'un masque et d'un ballon anesthésique, employés en anesthésie pédiatrique, permet lorsque les voies respiratoires sont libres, d'éviter l'intubation avec ses risques minimes. Bien entendu l'intubation sera pratiquée si nécessaire. La ventilation au moyen du masque, bien contrôlée dans des conditions adéquates, permet d'éviter l'hypoxie du nouveau-né et ses conséquences redoutables.

Lorsque au cours du travail on pouvait prévoir un risque de dépression respiratoire chez le nouveau-né, un second appareil d'anesthésie était apporté dans la salle d'accouchement qu'il fallait brancher à une autre source d'oxygène afin d'y relier le masque ou le système d'intubation pédiatrique (Fig. 1). Ceci n'aidait pas à corriger les problèmes d'encombrement. De plus, lorsque le système de canalisation d'oxygène est distribué aux deux extrémités de la salle, l'anesthésiste devait quitter la patiente, la laissant s'éveiller et la confiant à la surveillance d'une infirmière pour s'occuper lui-même du nouveau-né, technique qui est loin d'étre idéale.

Il est possible de parer à ces inconvénients en fermant les gaz anesthésiques de l'appareil employé par la mère et en utilisant la source unique d'oxygène pour l'enfant, tout en surveillant la mère, qu'il faudra peut être endormir de nouveau, une fois le bébé en bon état. Pratique qui n'est pas sans danger.

Nous avons imaginé pouvoir pallier aux difficultés citées plus haut, d'une façon simple et peu couteuse, par l'adjonction d'un second débitmètre sur la canalisation d'oxygène de l'appareil anesthésique. De cette façon un seul appareil anesthésique peut assurer l'administration d'agents anesthésiques à la mère tout en permettant d'oxygéner le nouveau-né. Technique de choix dans les circonstances.

L'anesthésiste peut s'occuper rapidement d'oxygéner le nouveau-né dès que les voies respiratoires ont été dégagées, en le ventilant avec un masque pédiatrique. Si la technique n'est pas efficaces l'intubation est pratiquée, le matériel à cet effet étant disponible en tout temps.

C'est ici que notre nouveau dispositif prouve son utilité. La source d'oxygène du débitmètre ajouté permet de disposer de débit connu, l'anesthésiste rompu a la ventilation du jeune enfant peut apprécier le degré de pression qu'il exerce

"Hôpital Ste. Justine, Montréal, P.Q. 


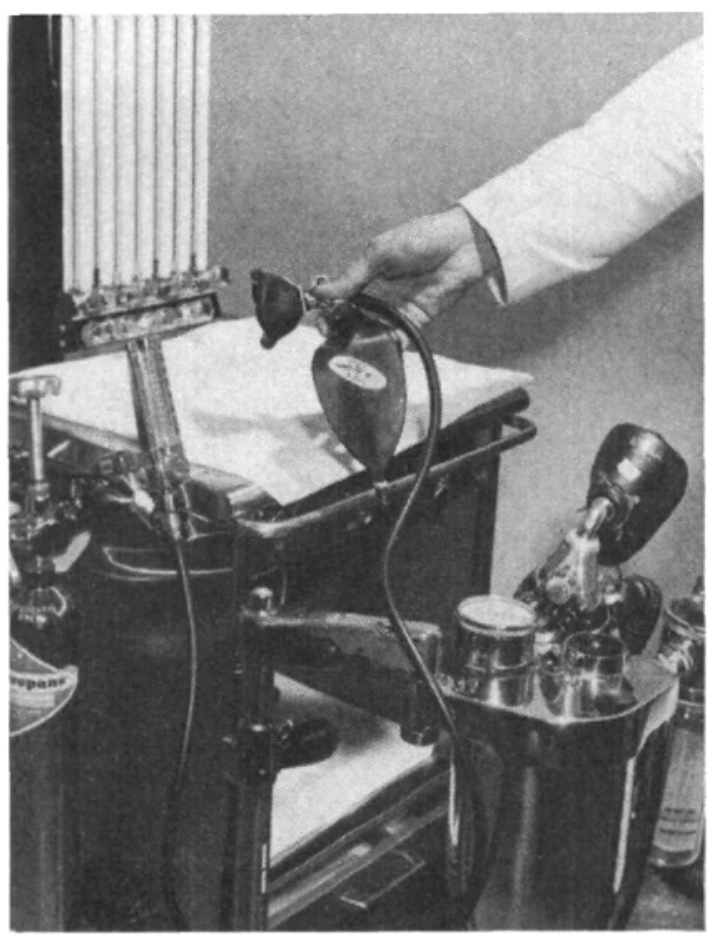

Figure 1. La disposition du second debimètre, relié à un masque d'un ballon pédiatrique. Le masque peut être remplacé rapidement par un raccord endotrachéal.

sur le ballon et un manomètre pourrait être relié au dispositif de ventilation permettant d'éviter l'emploi de pression excessive.

Cette modification simple des appareils anesthésiques en usage permet d'agir rapidement en attendant du renfort ou si livré seul à son sort l'anesthésiste, peut entreprendre la réanimation du bébé sans négliger la surveillance de la mère.

En pratique il est souvent difficile de disposer de deux anesthésistes pour chaque cas d'obstétrique. La mise en place des instruments nécessaires à la réanimation évite la perte de secondes précieuses et facilite l'exercice de l'anesthésie en salle d'accouchement où souvent l'adresse et le sang-froid sont mis à rude épreuve.

De plus, le dispositif décrit plus haut est employé depuis plus d'un an a l'hôpital Sainte-Justine, il nous a rendu de grands services et nous n'avons eu qu'à nous en féliciter. Nous croyons que cette source d'oxygène supplémentaire peut trouver place partout où l'anesthésie obstétricale se pratique d la plus grande satisfaction de tous.

\section{RÉSUMÉ}

Cet article décrit l'addition d'un débitmètre à oxygène sur l'appareil employé en anesthésie obstétricale. Ce dispositif permet une ventilation rapide et efficace du nouveau-né montrant des signes de détresse respiratoire. 